\title{
DISCONTINUOUS GALERKIN METHOD FOR THE THREE-DIMENSIONAL PROBLEM OF THERMOELASTICITY
}

\author{
Ewa Wegrzyn-Skrzypczak \\ Institute of Mathematics, Czestochowa University of Technology \\ Czestochowa, Poland \\ ewa.skrzypczak@im.pcz.pl
}

Received: 14 October 2019; Accepted: 8 December 2019

\begin{abstract}
The paper is focused on the mathematical and numerical approaches for the thermoelasticity problem in the three-dimensional domain. The mathematical description of considered problem is based on the second order differential equations of elasticity with the term describing thermal deformations. The numerical model uses the discontinuous Galerkin method which is widely used to solve the problems of hydrodynamics. The presented paper shows the possibility of using the mentioned method to solve the problem of thermomechanics.
\end{abstract}

MSC 2010: 35J25, 65M60

Keywords: thermoelasticity, finite element method, discontinuous Galerkin method

\section{Introduction}

One of the reasons for the change of volume and shape of the bodies is temperature. Most often, the volume of the body increases as the temperature increases, e.g. a heated steel bar increases its dimensions. If the body, which is thermally loaded, is a free body then the change in volume does not generate stress. In the case of a non-free body, the imposed constraints make it impossible to change the volume freely, which results in stresses. If the stress level does not exceed the yield strength of the material the body is made of, then this is the problem of thermoelasticity.

Thermoelasticity relates to the analysis of elastic bodies exposed to the temperature. Additional loads may be forces or pressure. In the general case, the mechanical and thermal loads are mutually coupled.

One of the numerical methods used to solve differential equations describing many physical phenomena is the discontinuous Galerkin method (DGM). DGM is a method that has been widely described in literature since the 1970 s, its history and basics are most widely described in [1]. However, there are not many papers 
on its application in the issues of linear elasticity. DGM in the theory of linear elasticity develops in two directions. The first direction of development includes the use of LDG (Local Discontinuous Galerkin) and IPDG (Interior Penalty Discontinuous Galerkin) [2-6]. The second direction of development, MDG (Mixed Discontinuous Galerkin) is based on the use of so-called mixed elements containing two base spaces of functions with different orders - lower for approximation of displacements and higher for approximation of stresses $[7,8]$.

\section{Mathematical model}

Let's consider a three-dimensional region $\Omega$ presented in Figure 1. Displacements $u(x, y, z)$ of the selected part of external surface are considered as given. The temperature distribution $T(x, y, z)$ in the whole region is also known.

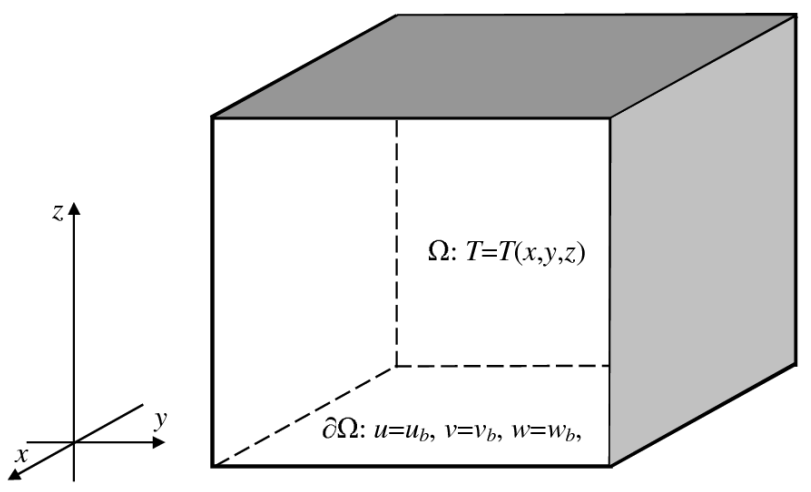

Fig. 1. Considered volume $\Omega$ with the temperature field $T(x, y, z)$ and displacements

$$
u(x, y, z) \text { on the bottom surface } \partial \Omega
$$

The main goal is to obtain the solution in the form of components of the displacement vector. The starting point of consideration is the system of equilibrium equations:

$$
\begin{gathered}
\frac{\partial \sigma_{x}}{\partial x}+\frac{\partial \tau_{x y}}{\partial y}+\frac{\partial \tau_{x z}}{\partial z}=0 \quad \frac{\partial \tau_{x y}}{\partial x}+\frac{\partial \sigma_{y}}{\partial y}+\frac{\partial \tau_{y z}}{\partial z}=0 \\
\frac{\partial \tau_{x z}}{\partial x}+\frac{\partial \tau_{y z}}{\partial y}+\frac{\partial \sigma_{z}}{\partial z}=0
\end{gathered}
$$

where $\sigma_{x}, \sigma_{y}, \sigma_{z}$ are normal stresses, $\tau_{x y}=\tau_{y x}, \tau_{x z}=\tau_{z x}, \tau_{y z}=\tau_{z y}$ are shear stresses. The use of stresses in the equations (1) is not convenient to consider boundary conditions. Therefore, equations (1) will be transformed in the next chapter into so-called displacement form. 


\section{Numerical model}

The discontinuous Galerkin method is derived from the criterion of the weighted residuals method, where the differential equation is multiplied by the weight function and integrated over the considered domain. The integration is performed in a single finite element. This involves the necessity of taking into account the boundary integrals of the so-called numerical fluxes in each finite element.

The first stage of the DG method is spatial discretization of the considered domain. In this case tetrahedral elements are used (Fig. 2).

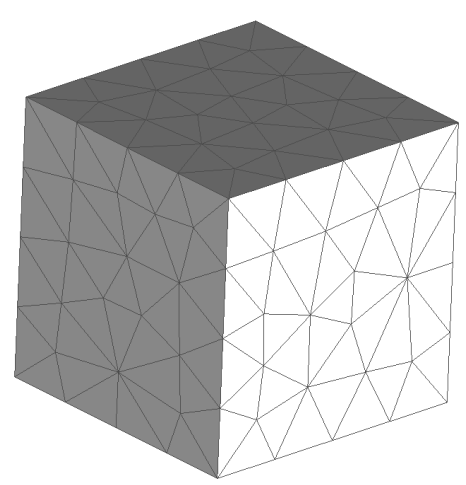

Fig. 2. Spatially discretized domain

Assume that the domain $\Omega$ consists of $N$ tetrahedrons:

$$
\Omega=\bigcup_{j=1}^{N} \Omega_{j}
$$

Using the criterion of the weighted residuals method for $j$-th element equations (1) are multiplied by the weighting function $\phi$ and integrated over the volume $\Omega_{j}$ :

$$
\begin{gathered}
\int_{\Omega_{j}} \phi\left(\frac{\partial \sigma_{x}}{\partial x}+\frac{\partial \tau_{x y}}{\partial y}+\frac{\partial \tau_{x z}}{\partial z}\right) d V=0 \quad \int_{\Omega_{j}} \phi\left(\frac{\partial \tau_{x y}}{\partial x}+\frac{\partial \sigma_{y}}{\partial y}+\frac{\partial \tau_{y z}}{\partial z}\right) d V=0 \\
\int_{\Omega_{j}} \phi\left(\frac{\partial \tau_{x z}}{\partial x}+\frac{\partial \tau_{y z}}{\partial y}+\frac{\partial \sigma_{z}}{\partial z}\right) d V=0
\end{gathered}
$$

The following relationships between stresses and strains are used:

$$
\begin{array}{lc}
\sigma_{x}=\left[2 f_{3} \varepsilon_{x}+f_{2}\left(\varepsilon_{x}+\varepsilon_{y}+\varepsilon_{z}\right)-f_{4} \varepsilon_{T}\right] & \tau_{x y}=\tau_{y x}=f_{3} \gamma_{x y} \\
\sigma_{y}=\left[2 f_{3} \varepsilon_{y}+f_{2}\left(\varepsilon_{x}+\varepsilon_{y}+\varepsilon_{z}\right)-f_{4} \varepsilon_{T}\right] & \tau_{y z}=\tau_{z y}=f_{3} \gamma_{y z} \\
\sigma_{z}=\left[2 f_{3} \varepsilon_{z}+f_{2}\left(\varepsilon_{x}+\varepsilon_{y}+\varepsilon_{z}\right)-f_{4} \varepsilon_{T}\right] & \tau_{x z}=\tau_{z x}=f_{3} \gamma_{x z}
\end{array}
$$


where $\varepsilon_{x}, \varepsilon_{y}, \varepsilon_{z}$ are linear strains, $\gamma_{x y}, \gamma_{y z}, \gamma_{x z}$ represent angular strains, $\varepsilon_{T}$ is a thermal strain, $f_{1}, f_{2}, f_{3}, f_{4}$ are the coefficients of elasticity listed in Table 1.

Table 1. Coefficients of elasticity

\begin{tabular}{|l|l|}
\hline$f_{1}$ & $\frac{E(1-v)}{(1+v)(1-2 v)}$ \\
\hline$f_{2}$ & $\frac{E v}{(1+v)(1-2 v)}$ \\
\hline$f_{3}$ & $\frac{E}{2(1+v)}$ \\
\hline$f_{4}$ & $\frac{E(1+v)}{(1+v)(1-2 v)}$ \\
\hline
\end{tabular}

The above coefficients depend on the Young's modulus $E\left[\mathrm{~N} / \mathrm{m}^{2}\right]$ and Poisson's ratio $v[-]$. Additionally the following geometrical relationships are used:

$$
\begin{array}{ll}
\varepsilon_{T}=\alpha \Delta T & \\
\varepsilon_{x}=\frac{\partial u}{\partial x}, & \gamma_{x y}=\gamma_{y x}=\frac{\partial u}{\partial y}+\frac{\partial v}{\partial x} \\
\varepsilon_{y}=\frac{\partial v}{\partial y}, & \gamma_{y z}=\gamma_{z y}=\frac{\partial v}{\partial z}+\frac{\partial w}{\partial y} \\
\varepsilon_{z}=\frac{\partial w}{\partial z}, & \gamma_{x z}=\gamma_{z x}=\frac{\partial u}{\partial z}+\frac{\partial w}{\partial x}
\end{array}
$$

where $u, v, w[\mathrm{~m}]$ are the components of the displacement vector, $\Delta T[\mathrm{~K}]$ represents the temperature difference between the reference and current temperatures in the considered body, $\alpha\left[\mathrm{K}^{-1}\right]$ is a linear coefficient of thermal expansion.

Using geometrical relationships (5) in equations (4), the following displacement dependent form of stresses are obtained:

$$
\begin{array}{ll}
\sigma_{x}=f_{1} \frac{\partial u}{\partial x}+f_{2} \frac{\partial v}{\partial y}+f_{2} \frac{\partial w}{\partial z}-f_{4} \alpha \Delta T & \tau_{x y}=\tau_{y x}=f_{3}\left(\frac{\partial u}{\partial y}+\frac{\partial v}{\partial x}\right) \\
\sigma_{y}=f_{2} \frac{\partial u}{\partial x}+f_{1} \frac{\partial v}{\partial y}+f_{2} \frac{\partial w}{\partial z}-f_{4} \alpha \Delta T & \tau_{y z}=\tau_{z y}=f_{3}\left(\frac{\partial v}{\partial z}+\frac{\partial w}{\partial y}\right) \\
\sigma_{z}=f_{2} \frac{\partial u}{\partial x}+f_{2} \frac{\partial v}{\partial y}+f_{1} \frac{\partial w}{\partial z}-f_{4} \alpha \Delta T & \tau_{x z}=\tau_{z x}=f_{3}\left(\frac{\partial u}{\partial z}+\frac{\partial w}{\partial x}\right)
\end{array}
$$

Substituting stresses in equations (3) by the relations (6), one obtains the displacement dependent integral form of thermoelasticity equations: 


$$
\begin{aligned}
& \int_{\Omega_{j}} \phi\left[\frac{\partial}{\partial x}\left(f_{1} \frac{\partial u}{\partial x}+f_{2} \frac{\partial v}{\partial y}+f_{2} \frac{\partial w}{\partial z}-f_{4} \alpha \Delta T\right)+f_{3} \frac{\partial}{\partial y}\left(\frac{\partial u}{\partial y}+\frac{\partial v}{\partial x}\right)+f_{3} \frac{\partial}{\partial z}\left(\frac{\partial u}{\partial z}+\frac{\partial w}{\partial x}\right)\right] d V=0 \\
& \int_{\Omega_{j}} \phi\left[f_{3} \frac{\partial}{\partial x}\left(\frac{\partial u}{\partial y}+\frac{\partial v}{\partial x}\right)+\frac{\partial}{\partial y}\left(f_{2} \frac{\partial u}{\partial x}+f_{1} \frac{\partial v}{\partial y}+f_{2} \frac{\partial w}{\partial z}-f_{4} \alpha \Delta T\right)+f_{3} \frac{\partial}{\partial z}\left(\frac{\partial v}{\partial z}+\frac{\partial w}{\partial y}\right)\right] d V=0 \\
& \int_{\Omega_{j}} \phi\left[f_{3} \frac{\partial}{\partial x}\left(\frac{\partial u}{\partial z}+\frac{\partial w}{\partial x}\right)+f_{3} \frac{\partial}{\partial y}\left(\frac{\partial v}{\partial z}+\frac{\partial w}{\partial y}\right)+\frac{\partial}{\partial z}\left(f_{2} \frac{\partial u}{\partial x}+f_{2} \frac{\partial v}{\partial y}+f_{1} \frac{\partial w}{\partial z}-f_{4} \alpha \Delta T\right)\right] d V=0
\end{aligned}
$$

Unfortunately, the DG method is not suitable for the second order differential equations. Therefore, in order to lower the order of equations (7), additional variables are introduced:

$$
\begin{array}{lll}
q_{x}^{(u)}=\frac{\partial u}{\partial x}, & q_{y}^{(u)}=\frac{\partial u}{\partial y}, & q_{z}^{(u)}=\frac{\partial u}{\partial z} \\
q_{x}^{(v)}=\frac{\partial v}{\partial x} & q_{y}^{(v)}=\frac{\partial v}{\partial y} & q_{z}^{(v)}=\frac{\partial v}{\partial z} \\
q_{x}^{(w)}=\frac{\partial w}{\partial x} & q_{y}^{(w)}=\frac{\partial w}{\partial y} & q_{z}^{(w)}=\frac{\partial w}{\partial z}
\end{array}
$$

After using the relation (8) in equations (7) one can write:

$$
\begin{aligned}
& \int_{\Omega_{j}} \phi\left[\frac{\partial}{\partial x}\left(f_{1} q_{x}^{(u)}+f_{2} q_{y}^{(v)}+f_{2} q_{z}^{(w)}-f_{4} \alpha \Delta T\right)+f_{3} \frac{\partial}{\partial y}\left(q_{y}^{(u)}+q_{x}^{(v)}\right)+f_{3} \frac{\partial}{\partial z}\left(q_{z}^{(u)}+q_{x}^{(w)}\right)\right] d V=0 \\
& \int_{\Omega_{j}} \phi\left[f_{3} \frac{\partial}{\partial x}\left(q_{y}^{(u)}+q_{x}^{(v)}\right)+\frac{\partial}{\partial y}\left(f_{2} q_{x}^{(u)}+f_{1} q_{y}^{(v)}+f_{2} q_{z}^{(w)}-f_{4} \alpha \Delta T\right)+f_{3} \frac{\partial}{\partial z}\left(q_{z}^{(v)}+q_{y}^{(w)}\right)\right] d V=0 \\
& \int_{\Omega_{j}} \phi\left[f_{3} \frac{\partial}{\partial x}\left(q_{z}^{(u)}+q_{x}^{(w)}\right)+f_{3} \frac{\partial}{\partial y}\left(q_{z}^{(v)}+q_{y}^{(w)}\right)+\frac{\partial}{\partial z}\left(f_{2} q_{x}^{(u)}+f_{2} q_{y}^{(v)}+f_{1} q_{z}^{(w)}-f_{4} \alpha \Delta T\right)\right] d V=0
\end{aligned}
$$

The criterion of the weighted residuals method is also applied to equations (8):

$$
\begin{aligned}
& \int_{\Omega_{j}} \phi q_{x}^{(u)} d V=\int_{\Omega_{j}} \phi \frac{\partial u}{\partial x} d V, \quad \int_{\Omega_{j}} \phi q_{y}^{(u)} d V=\int_{\Omega_{j}} \phi \frac{\partial u}{\partial y} d V, \quad \int_{\Omega_{j}} \phi q_{z}^{(u)} d V=\int_{\Omega_{j}} \phi \frac{\partial u}{\partial z} d V \\
& \int_{\Omega_{j}} \phi q_{x}^{(v)} d V=\int_{\Omega_{j}} \phi \frac{\partial v}{\partial x} d V, \quad \int_{\Omega_{j}} \phi q_{y}^{(v)} d V=\int_{\Omega_{j}} \phi \frac{\partial v}{\partial y} d V, \quad \int_{\Omega_{j}} \phi q_{z}^{(v)} d V=\int_{\Omega_{j}} w \frac{\partial v}{\partial z} d V \\
& \int_{\Omega_{j}} \phi q_{x}^{(w)} d V=\int_{\Omega_{j}} \phi \frac{\partial w}{\partial x} d V, \quad \int_{\Omega_{j}} \phi q_{y}^{(w)} d V=\int_{\Omega_{j}} \phi \frac{\partial w}{\partial y} d V, \quad \int_{\Omega_{j}} \phi q_{z}^{(w)} d V=\int_{\Omega_{j}} \phi \frac{\partial w}{\partial z} d V
\end{aligned}
$$


Equations (9)-(10) are the set of twelve equations with twelve unknowns which should be supplemented by the Dirichlet (11) and Neumann's boundary conditions (12) respectively:

$$
\begin{gathered}
(x, y, z) \in \partial \Omega: u=u_{b}, v=v_{b}, w=w_{b} \\
(x, y, z) \in \partial \Omega: q_{x}^{(u)}=q_{x b}^{(u)}, q_{y}^{(u)}=q_{y b}^{(u)}, q_{z}^{(u)}=q_{z b}^{(u)} \\
(x, y, z) \in \partial \Omega: q_{x}^{(v)}=q_{x b}^{(v)}, q_{y}^{(v)}=q_{y b}^{(v)}, q_{z}^{(v)}=q_{z b}^{(v)} \\
(x, y, z) \in \partial \Omega: q_{x}^{(w)}=q_{x b}^{(w)}, q_{y}^{(w)}=q_{y b}^{(w)}, q_{z}^{(w)}=q_{z b}^{(w)}
\end{gathered}
$$

The application of the DG method is presented for the first equation from the set (10). Operations for the other equations are done in the same way. The integration by parts is used and $q_{x}^{(u)}$ and $u$ are replaced by the approximation $q_{x h}^{(u)}$ and $u_{h}$ :

$$
\int_{\Omega_{j}} \phi q_{x h}^{(u)} d V=-\int_{\Omega_{j}} \frac{\partial \varphi}{\partial x} u_{h} d V+\int_{\partial \Omega_{j}} \phi n_{x} \hat{u}_{h} d s
$$

where $n_{x}$ is the component of the vector perpendicular to the surface of the finite element, whereas, $q_{x h}^{(u)}$ and $u_{h}$ are expressed as follows:

$$
\begin{gathered}
q_{x h}^{(u)}=\sum_{i=1}^{m} \varphi_{i} q_{x i}^{(u)}=\boldsymbol{\Phi}^{T} \mathbf{q}_{x}^{(u)} \\
u_{h}=\sum_{i=1}^{m} \varphi_{i} u_{i}=\boldsymbol{\Phi}^{T} \mathbf{u}
\end{gathered}
$$

where $\varphi_{i}$ is the $i$-th shape function of the finite element, $m$ is the number of nodes in the finite element depending on the order of approximation $p$ :

$$
m=\frac{(p+1)(p+2)(p+3)}{6}
$$

To simplify further considerations, the weight functions are assumed the same as the shape functions $\phi=\varphi$. The quantity $\hat{u}_{h}$ in equation (13) is the so-called numerical flux, which is defined in literature in various ways [9]. In the presented description the so-called central flux is used:

$$
\hat{u}_{h}=\frac{1}{2}\left(u_{h}^{+}+u_{h}^{-}\right)
$$

where "+" and "-“" are boundary values calculated in $j$-th finite element and adjacent elements respectively. 
According to the presented assumptions, equation (13) can be written as follows:

$$
\int_{\Omega_{j}} \phi q_{x h}^{(u)} d V=-\int_{\Omega_{j}} \frac{\partial \varphi}{\partial x} u_{h} d V+\int_{\partial \Omega_{j}} \phi n_{x} \frac{1}{2}\left(u_{h}^{+}+u_{h}^{-}\right) d s
$$

The quantities $q_{x h}^{(u)}, u_{h}, u_{h}^{+}$are assumed unknown, while $u_{h}^{-}$is given. Therefore, equation (18) should be rearranged, so that the elements containing the unknown terms are on the right, while the rest are moved on the left:

$$
\int_{\Omega_{j}} \phi q_{x h}^{(u)} d V+\int_{\Omega_{j}} \frac{\partial \varphi}{\partial x} u_{h} d V-\int_{\partial \Omega_{j}} \phi n_{x} \frac{1}{2} u_{h}^{+} d s=\int_{\partial \Omega_{j}} \phi n_{x} \frac{1}{2} u_{h}^{-} d s
$$

The rest of equations from the set (10) are obtained in the same way. The equations from the set (9) must be also rearranged in order to satisfy DG assumption The first equation from (9) is taken as an example:

$$
\int_{\Omega_{j}} \varphi\left[\frac{\partial}{\partial x}\left(f_{1} q_{x}^{(u)}+f_{2} q_{y}^{(v)}+f_{2} q_{z}^{(w)}-f_{4} \alpha \Delta T\right)+f_{3} \frac{\partial}{\partial y}\left(q_{y}^{(u)}+q_{x}^{(v)}\right)+f_{3} \frac{\partial}{\partial z}\left(q_{z}^{(u)}+q_{x}^{(w)}\right)\right] d V=0
$$

After replacing the exact solution $q_{x}^{(u)}$ by approximation $q_{x h}^{(u)}$, the first term in (20) is written as follows:

$$
\int_{\Omega_{j}} \phi \frac{\partial}{\partial x}\left(f_{1} q_{x h}^{(u)}\right) d V=-\int_{\Omega_{j}} f_{1} \frac{\partial \varphi}{\partial x} q_{x h}^{(u)} d V+\int_{\partial \Omega_{j}} f_{1} \phi n_{x} \hat{q}_{x h}^{(u)} d s
$$

According to [9], the central stream $\hat{q}_{x h}^{(u)}$ equals:

$$
\hat{q}_{x h}^{(u)}=\frac{1}{2}\left(q_{x h}^{(u)+}+q_{x h}^{(u)-}\right)-C_{11}\left(n_{x}^{+} u_{h}^{+}+n_{x}^{-} u_{h}^{-}\right)
$$

where $C_{11}$ is a constant providing convergence of the solution whose value is chosen experimentally.

Using relation (22) in equation (21) one can write:

$$
\int_{\Omega_{j}} \phi \frac{\partial}{\partial x}\left(f_{1} q_{x h}^{(u)}\right) d V=-\int_{\Omega_{j}} f_{1} \frac{\partial \phi}{\partial x} q_{x h}^{(u)} d V+\int_{\partial \Omega_{j}} f_{1} \phi n_{x}\left[\frac{1}{2}\left(q_{x h}^{(u)+}+q_{x h}^{(u)-}\right)-C_{11}\left(n_{x}^{+} u_{h}^{+}+n_{x}^{-} u_{h}^{-}\right)\right] d s
$$


122

E. Węgrzyn-Skrzypczak

The equation (23) can be written as the sum of integrals:

$$
\begin{aligned}
& \int_{\Omega_{j}} \phi \frac{\partial}{\partial x}\left(f_{1} q_{x h}^{(u)}\right) d V=-\int_{\Omega_{j}} f_{1} \frac{\partial \phi}{\partial x} q_{x h}^{(u)} d V+\int_{\partial \Omega_{j}} f_{1} \phi n_{x} \frac{1}{2} q_{x h}^{(u)+} d s+ \\
& +\int_{\partial \Omega_{j}} f_{1} \phi n_{x} \frac{1}{2} q_{x h}^{(u)-} d s-\int_{\partial \Omega_{j}} f_{1} \phi n_{x} C_{11} n_{x}^{+} u_{h}^{+} d s-\int_{\partial \Omega_{j}} f_{1} \phi n_{x} C_{11} n_{x}^{-} u_{h}^{-} d s
\end{aligned}
$$

Assuming that $n_{x} n_{x}^{+}=n_{x}^{2}, n_{x} n_{x}^{-}=-n_{x}^{2}$ one can write:

$$
\begin{aligned}
& \int_{\Omega_{j}} \phi \frac{\partial}{\partial x}\left(f_{1} q_{x h}^{(u)}\right) d V=-\int_{\Omega_{j}} f_{1} \frac{\partial \phi}{\partial x} q_{x h}^{(u)} d V+\int_{\partial \Omega_{j}} f_{1} \phi n_{x} \frac{1}{2} q_{x h}^{(u)+} d s+ \\
& +\int_{\partial \Omega_{j}} f_{1} \phi n_{x} \frac{1}{2} q_{x h}^{(u)-} d s-\int_{\partial \Omega_{j}} f_{1} \phi n_{x}^{2} C_{11} u_{h}^{+} d s+\int_{\partial \Omega_{j}} f_{1} \phi n_{x}^{2} C_{11} u_{h}^{-} d s
\end{aligned}
$$

Other terms appearing in equation (20) are determined analogically:

$$
\begin{aligned}
& \int_{\Omega_{j}} \phi \frac{\partial}{\partial x}\left(f_{2} q_{y h}^{(v)}\right) d V=-\int_{\Omega_{j}} f_{2} \frac{\partial \phi}{\partial x} q_{y h}^{(v)} d V+\int_{\partial \Omega_{j}} f_{2} \phi n_{x} \frac{1}{2} q_{y h}^{(v)+} d s+ \\
& +\int_{\partial \Omega_{j}} f_{2} \phi n_{x} \frac{1}{2} q_{y h}^{(v)-} d s-\int_{\partial \Omega_{j}} f_{2} \phi n_{x} n_{y} C_{11} v_{h}^{+} d s+\int_{\partial \Omega_{j}} f_{2} \phi n_{x} n_{y} C_{11} v_{h}^{-} d s \\
& \int_{\Omega_{j}} \phi \frac{\partial}{\partial x}\left(f_{2} q_{z h}^{(w)}\right) d V=-\int_{\Omega_{j}} f_{2} \frac{\partial \phi}{\partial x} q_{z h}^{(w)} d V+\int_{\partial \Omega_{j}} f_{2} \phi n_{x} \frac{1}{2} q_{z h}^{(w)++} d s+ \\
& +\int_{\partial \Omega_{j}} f_{2} \phi n_{x} \frac{1}{2} q_{z h}^{(w)-} d s-\int_{\partial \Omega_{j}} f_{2} \phi n_{x} n_{z} C_{11} w_{h}^{+} d s+\int_{\partial \Omega_{j}} f_{2} \phi n_{x} n_{z} C_{11} w_{h}^{-} d s \\
& \quad \int_{\Omega_{j}} \phi \frac{\partial}{\partial y}\left(f_{3} q_{y h}^{(u)}\right) d V=-\int_{\Omega_{j}} f_{3} \frac{\partial \phi}{\partial y} q_{y h}^{(u)} d V+\int_{\partial \Omega_{j}} f_{3} \phi n_{y} \frac{1}{2} q_{y h}^{(u)+} d s+ \\
& +\int_{\partial \Omega_{j}} f_{3} \phi n_{y} \frac{1}{2} q_{y h}^{(u)-} d s-\int_{\partial \Omega_{j}} f_{3} \phi n_{y}^{2} C_{11} u_{h}^{+} d s+\int_{\partial \Omega_{j}} f_{3} \phi n_{y}^{2} C_{11} u_{h}^{-} d s \\
& \int_{\Omega_{j}} \phi \frac{\partial}{\partial y}\left(f_{3} q_{x h}^{(v)}\right) d V=-\int_{\Omega_{j}} f_{3} \frac{\partial \phi}{\partial y} q_{x h}^{(v)} d V+\int_{\partial \Omega_{j}} f_{3} \phi n_{y} \frac{1}{2} q_{x h}^{(v)+} d s+ \\
& +\int_{\partial \Omega_{j}} f_{3} \phi n_{y} \frac{1}{2} q_{x h}^{(v)-} d s-\int_{\partial \Omega_{j}} f_{3} \phi n_{y} n_{x} C_{11} v_{h}^{+} d s+\int_{\partial \Omega_{j}} f_{3} \phi n_{y} n_{x} C_{11} v_{h}^{-} d s
\end{aligned}
$$


Discontinuous Galerkin method for the three-dimensional problem of thermoelasticity

123

$$
\begin{aligned}
& \int_{\Omega_{j}} \phi \frac{\partial}{\partial z}\left(f_{3} q_{z h}^{(u)}\right) d V=-\int_{\Omega_{j}} f_{3} \frac{\partial \phi}{\partial z} q_{z h}^{(u)} d V+\int_{\partial \Omega_{j}} f_{3} \phi n_{z} \frac{1}{2} q_{z h}^{(u)+} d s+ \\
& +\int_{\partial \Omega_{j}} f_{3} \phi n_{z} \frac{1}{2} q_{z h}^{(u)-} d s-\int_{\partial \Omega_{j}} f_{3} \phi n_{z}^{2} C_{11} u_{h}^{+} d s+\int_{\partial \Omega_{j}} f_{3} \phi n_{z}^{2} C_{11} u_{h}^{-} d s \\
& \int_{\Omega_{j}} \phi \frac{\partial}{\partial z}\left(f_{3} q_{x h}^{(w)}\right) d V=-\int_{\Omega_{j}} f_{3} \frac{\partial \phi}{\partial z} q_{x h}^{(w)} d V+\int_{\partial \Omega_{j}} f_{3} \phi n_{z} \frac{1}{2} q_{x h}^{(w)+} d s+ \\
& +\int_{\partial \Omega_{j}} f_{3} \phi n_{z} \frac{1}{2} q_{x h}^{(w)-} d s-\int_{\partial \Omega_{j}} f_{3} \phi n_{z} n_{x} C_{11} w_{h}^{+} d s+\int_{\partial \Omega_{j}} f_{3} \phi n_{z} n_{x} C_{11} w_{h}^{-} d s \\
& \int_{\Omega_{j}} \phi \frac{\partial}{\partial x}\left(f_{4} \alpha \Delta T_{h}\right) d V=-\int_{\Omega_{j}} f_{4} \alpha \frac{\partial \phi}{\partial x} \Delta T_{h} d V+\int_{\partial \Omega_{j}} f_{4} \alpha \phi n_{x} \frac{1}{2} \Delta T_{h} d s
\end{aligned}
$$

Using relations (25)-(32) in equation (20) it can be written:

$$
\begin{aligned}
& -f_{1} \int_{\Omega_{j}} \frac{\partial \phi}{\partial x} q_{x h}^{(u)} d V-f_{3} \int_{\Omega_{j}} \frac{\partial \phi}{\partial y} q_{y h}^{(u)} d V-f_{3} \int_{\Omega_{j}} \frac{\partial \phi}{\partial z} q_{z h}^{(u)} d V-f_{3} \int_{\Omega_{j}} \frac{\partial \phi}{\partial y} q_{x h}^{(v)} d V+ \\
& -f_{2} \int_{\Omega_{j}} \frac{\partial \phi}{\partial x} q_{y h}^{(v)} d V-f_{3} \int_{\Omega_{j}} \frac{\partial \phi}{\partial z} q_{x h}^{(w)} d V-f_{2} \int_{\Omega_{j}} \frac{\partial \phi}{\partial x} q_{z h}^{(w)} d V+ \\
& +\frac{1}{2} f_{1} n_{x} \int_{\partial \Omega_{j}} \phi q_{x h}^{(u)+} d s+\frac{1}{2} f_{3} n_{y} \int_{\partial \Omega_{j}} \phi q_{y h}^{(u)+} d s+\frac{1}{2} f_{3} n_{z} \int_{\partial \Omega_{j}} \phi q_{z h}^{(u)+} d s+\frac{1}{2} f_{3} n_{y} \int_{\partial \Omega_{j}} \phi q_{x h}^{(v)+} d s+ \\
& +\frac{1}{2} f_{2} n_{x} \int_{\partial \Omega_{j}} \phi q_{y h}^{(v)+} d s+\frac{1}{2} f_{3} n_{z} \int_{\partial \Omega_{j}} \phi q_{x h}^{(w)+} d s+\frac{1}{2} f_{2} n_{x} \int_{\partial \Omega_{j}} \phi q_{z h}^{(w)+} d s+ \\
& -C_{11}\left(f_{1} n_{x}^{2}+f_{3} n_{y}^{2}+f_{3} n_{z}^{2}\right) \int_{\partial \Omega_{j}} \phi u_{h}^{+} d s-C_{11}\left(f_{3}+f_{2}\right) n_{y} n_{x} \int_{\partial \Omega_{j}} \phi v_{h}^{+} d s+ \\
& -C_{11}\left(f_{3}+f_{2}\right) n_{z} n_{x} \int_{\partial \Omega_{j}} \phi w_{h}^{+} d s=\frac{1}{2} f_{1} n_{x} \int_{\partial \Omega_{j}} \phi q_{x h}^{(u)-} d s+\frac{1}{2} f_{3} n_{y} \int_{\partial \Omega_{j}} \phi q_{y h}^{(u)-} d s+ \\
& +\frac{1}{2} f_{3} n_{z} \int_{\partial \Omega_{j}} \phi q_{z h}^{(u)-} d s+\frac{1}{2} f_{3} n_{y} \int_{\partial \Omega_{j}} \phi q_{x h}^{(v)-} d s++\frac{1}{2} f_{2} n_{x} \int_{\partial \Omega_{j}} \phi q_{y h}^{(v)-} d s+\frac{1}{2} f_{3} n_{z} \int_{\partial \Omega_{j}} \phi q_{x h}^{(w)-} d s+ \\
& +\frac{1}{2} f_{2} n_{x} \int_{\partial \Omega_{j}} \phi q_{z h}^{(w)-} d s-C_{11}\left(f_{1} n_{x}^{2}+f_{3} n_{y}^{2}+f_{3} n_{z}^{2}\right) \int_{\partial \Omega_{j}} \phi u_{h}^{-} d s-C_{11}\left(f_{3}+f_{2}\right) n_{y} n_{x} \int_{\partial \Omega_{j}} \phi v_{h}^{-} d s+ \\
& -C_{11}\left(f_{3}+f_{2}\right) n_{z} n_{x} \int_{\partial \Omega_{j}} \phi w_{h}^{-} d s-f_{4} \alpha \int_{\Omega_{j}} \frac{\partial \phi}{\partial x} \Delta T_{h} d V+\frac{1}{2} f_{4} \alpha n_{x} \int_{\partial \Omega_{j}} \phi \Delta T_{h} d s
\end{aligned}
$$


The last two equations from the set (10) are derived analogously. The terms from the left side of the equations build the stiffness matrix, while the terms from the right form the vector of boundary values. The following matrices are introduced:

$$
\begin{aligned}
& \mathbf{M}=\int_{\Omega^{(e)}} \boldsymbol{\Phi} \boldsymbol{\Phi}^{T} d V \\
& \mathbf{D}_{x}=\int_{\Omega^{(e)}}\left(\frac{\partial \boldsymbol{\Phi}}{\partial x}\right) \boldsymbol{\Phi}^{T} d V-\mathbf{B}_{x}, \quad \mathbf{B}_{x}=\frac{1}{2} n_{x} \int_{\partial \Omega^{(e)}} \boldsymbol{\Phi} \boldsymbol{\Phi}^{T} d s \\
& \mathbf{D}_{y}=\int_{\Omega^{(e)}}\left(\frac{\partial \boldsymbol{\Phi}}{\partial y}\right) \mathbf{\Phi}^{T} d V-\mathbf{B}_{y}, \quad \mathbf{B}_{y}=\frac{1}{2} n_{y} \int_{\partial \Omega^{(e)}} \boldsymbol{\Phi}^{T} d s \\
& \mathbf{D}_{z}=\int_{\Omega^{(e)}}\left(\frac{\partial \boldsymbol{\Phi}}{\partial x}\right) \boldsymbol{\Phi}^{T} d V-\mathbf{B}_{z}, \quad \mathbf{B}_{z}=\frac{1}{2} n_{z} \int_{\partial \Omega^{(e)}} \boldsymbol{\Phi} \boldsymbol{\Phi}^{T} d s \\
& \mathbf{S}_{x x}=C_{11}\left(f_{1} n_{x}^{2}+f_{3} n_{y}^{2}+f_{3} n_{z}^{2}\right) \int_{\partial \Omega_{j}} \boldsymbol{\Phi} \boldsymbol{\Phi}^{T} d s \quad \mathbf{S}_{x y}^{(v)}=C_{11}\left(f_{3}+f_{2}\right) n_{y} n_{x} \int_{\partial \Omega_{j}} \boldsymbol{\Phi} \boldsymbol{\Phi}^{T} d s \\
& \mathbf{S}_{y y}^{(v)}=C_{11}\left(f_{3} n_{x}^{2}+f_{1} n_{y}^{2}+f_{3} n_{z}^{2}\right) \int_{\partial \Omega_{j}} \boldsymbol{\Phi} \boldsymbol{\Phi}^{T} d s \quad \mathbf{S}_{x z}^{(w)}=C_{11}\left(f_{3}+f_{2}\right) n_{z} n_{x} \int_{\partial \Omega_{j}} \boldsymbol{\Phi} \boldsymbol{\Phi}^{T} d s \\
& \mathbf{S}_{z z}^{(w)}=C_{11}\left(f_{3} n_{x}^{2}+f_{3} n_{y}^{2}+f_{1} n_{z}^{2}\right) \int_{\partial \Omega_{j}} \boldsymbol{\Phi}^{T} d s \quad \mathbf{S}_{y z}^{(w)}=C_{11}\left(f_{3}+f_{2}\right) n_{z} n_{y} \int_{\partial \Omega_{j}} \boldsymbol{\Phi} \boldsymbol{\Phi}^{T} d s \\
& \mathbf{H}_{x}=-f_{4} \alpha \int_{\Omega^{(e)}}\left(\frac{\partial \boldsymbol{\Phi}}{\partial x}\right) \boldsymbol{\Phi}^{T} \Delta \mathbf{T} d V-\mathbf{h}_{x}, \quad \mathbf{h}_{x}=\frac{1}{2} f_{4} \alpha n_{x} \int_{\partial \Omega^{(e)}} \boldsymbol{\Phi} \boldsymbol{\Phi}^{T} \Delta \mathbf{T} d s \\
& \mathbf{H}_{y}=-f_{4} \alpha \int_{\Omega^{(e)}}\left(\frac{\partial \boldsymbol{\Phi}}{\partial y}\right) \boldsymbol{\Phi}^{T} \Delta \mathbf{T} d V-\mathbf{h}_{y}, \quad \mathbf{h}_{y}=\frac{1}{2} f_{4} \alpha n_{y} \int_{\partial \Omega^{(e)}} \boldsymbol{\Phi} \boldsymbol{\Phi}^{T} \Delta \mathbf{T} d s \\
& \mathbf{H}_{z}=-f_{4} \alpha \int_{\Omega^{(e)}}\left(\frac{\partial \boldsymbol{\Phi}}{\partial z}\right) \boldsymbol{\Phi}^{T} \Delta \mathbf{T} d V-\mathbf{h}_{z}, \quad \mathbf{h}_{z}=\frac{1}{2} f_{4} \alpha n_{z} \int_{\partial \Omega^{(e)}} \boldsymbol{\Phi} \boldsymbol{\Phi}^{T} \Delta \mathbf{T} d s
\end{aligned}
$$

The above matrices and vectors are used to form the stiffness matrix $\mathbf{K}$ (38) and the vector $\mathbf{B}(42)$. The dimensions of $\mathbf{K}$ depend on the approximation order. In the case of linear approximation $p=2 \mathbf{K}$ contains 48 rows and columns. As $p$ increases, the matrix dimensions also increase rapidly, assuming $120 \mathrm{x} 120$ for $p=2$ and $240 \times 240$ for $p=3$. 
$\mathbf{K}=\left[\begin{array}{cccccccccccc}\mathbf{M} & \mathbf{0} & \mathbf{0} & \mathbf{0} & \mathbf{0} & \mathbf{0} & \mathbf{0} & \mathbf{0} & \mathbf{0} & \mathbf{D}_{x} & \mathbf{0} & \mathbf{0} \\ \mathbf{0} & \mathbf{M} & \mathbf{0} & \mathbf{0} & \mathbf{0} & \mathbf{0} & \mathbf{0} & \mathbf{0} & \mathbf{0} & \mathbf{D}_{y} & \mathbf{0} & \mathbf{0} \\ \mathbf{0} & \mathbf{0} & \mathbf{M} & \mathbf{0} & \mathbf{0} & \mathbf{0} & \mathbf{0} & \mathbf{0} & \mathbf{0} & \mathbf{D}_{z} & \mathbf{0} & \mathbf{0} \\ \mathbf{0} & \mathbf{0} & \mathbf{0} & \mathbf{M} & \mathbf{0} & \mathbf{0} & \mathbf{0} & \mathbf{0} & \mathbf{0} & \mathbf{0} & \mathbf{D}_{x} & \mathbf{0} \\ \mathbf{0} & \mathbf{0} & \mathbf{0} & \mathbf{0} & \mathbf{M} & \mathbf{0} & \mathbf{0} & \mathbf{0} & \mathbf{0} & \mathbf{0} & \mathbf{D}_{y} & \mathbf{0} \\ \mathbf{0} & \mathbf{0} & \mathbf{0} & \mathbf{0} & \mathbf{0} & \mathbf{M} & \mathbf{0} & \mathbf{0} & \mathbf{0} & \mathbf{0} & \mathbf{D}_{z} & \mathbf{0} \\ \mathbf{0} & \mathbf{0} & \mathbf{0} & \mathbf{0} & \mathbf{0} & \mathbf{0} & \mathbf{M} & \mathbf{0} & \mathbf{0} & \mathbf{0} & \mathbf{0} & \mathbf{D}_{x} \\ \mathbf{0} & \mathbf{0} & \mathbf{0} & \mathbf{0} & \mathbf{0} & \mathbf{0} & \mathbf{0} & \mathbf{M} & \mathbf{0} & \mathbf{0} & \mathbf{0} & \mathbf{D}_{y} \\ \mathbf{0} & \mathbf{0} & \mathbf{0} & \mathbf{0} & \mathbf{0} & \mathbf{0} & \mathbf{0} & \mathbf{0} & \mathbf{M} & \mathbf{0} & \mathbf{0} & \mathbf{D}_{z} \\ -f_{1} \mathbf{D}_{x}-f_{3} \mathbf{D}_{y} & -f_{3} \mathbf{D}_{z}-f_{3} \mathbf{D}_{y}-f_{2} \mathbf{D}_{x} & \mathbf{0} & -f_{3} \mathbf{D}_{z} & \mathbf{0} & -f_{2} \mathbf{D}_{x} & -\mathbf{S}_{x x} & -\mathbf{S}_{x y} & -\mathbf{S}_{x z} \\ -f_{2} \mathbf{D}_{y} & -f_{3} \mathbf{D}_{x} & \mathbf{0} & -f_{3} \mathbf{D}_{x} & -f_{1} \mathbf{D}_{y} & -f_{3} \mathbf{D}_{z} & \mathbf{0} & -f_{3} \mathbf{D}_{z} & -f_{2} \mathbf{D}_{y} & -\mathbf{S}_{x y} & -\mathbf{S}_{y y} & -\mathbf{S}_{y z} \\ -f_{2} \mathbf{D}_{z} & \mathbf{0} & -f_{3} \mathbf{D}_{x} & \mathbf{0} & -f_{2} \mathbf{D}_{z} & -f_{3} \mathbf{D}_{y} & -f_{3} \mathbf{D}_{x} & -f_{3} \mathbf{D}_{y} & -f_{1} \mathbf{D}_{z} & -\mathbf{S}_{x z} & -\mathbf{S}_{y z} & -\mathbf{S}_{z z}\end{array}\right]$

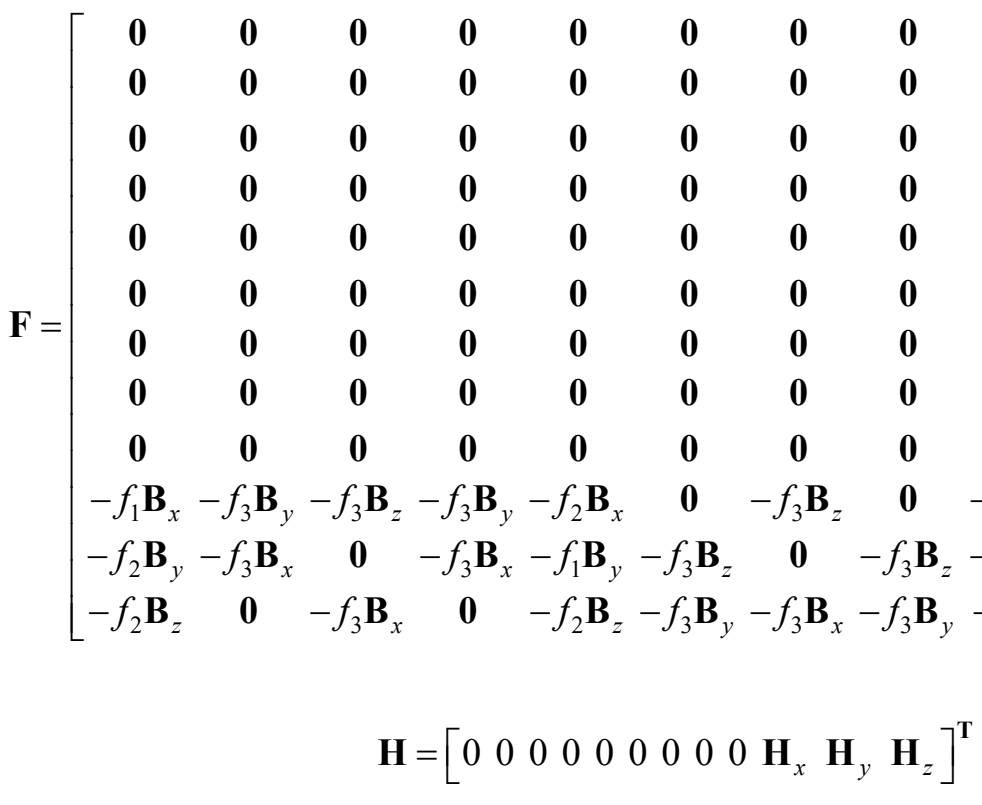

$$
\begin{aligned}
& \mathbf{y}=\left[\begin{array}{lllll}
\mathbf{q}_{x}^{(u)-} & \mathbf{q}_{y}^{(u)-} \mathbf{q}_{z}^{(u)-} \mathbf{q}_{x}^{(v)-} \mathbf{q}_{y}^{(v)-} \mathbf{q}_{z}^{(v)-} \mathbf{q}_{x}^{(w)-} \mathbf{q}_{y}^{(w)-} \mathbf{q}_{z}^{(w)-} \mathbf{u}^{-} \mathbf{v}^{-} \mathbf{w}^{-}
\end{array}\right]^{\mathbf{T}}
\end{aligned}
$$

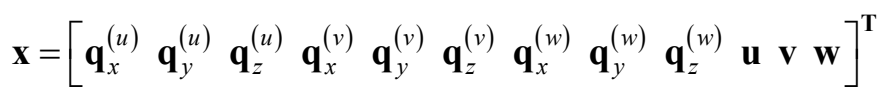

$$
\begin{aligned}
& \mathbf{B}=\mathbf{F} \mathbf{y}+\mathbf{H}
\end{aligned}
$$

Finally the set of equations can be written in the well-known form:

$$
\mathbf{K} \mathbf{x}=\mathbf{B}
$$


The calculation process must be done for each finite element. In each iteration it is necessary to solve $N$ systems of equations whose relative size is not large. The final solution is obtained after many iterations. $\mathbf{K}$ is built only once at the start of the process while $\mathbf{B}$ must be rebuilt in each iteration. Boundary conditions are introduced by the modification of appropriate values in the vector $\mathbf{y}$. During the iterative process the values from the boundaries are gradually propagated into the internal part of the domain. After each iteration, calculated nodal displacements are compared to the values obtained in the previous iteration. The calculation process is finished when the difference between nodal displacements from the two last iterations tends to zero. Obtained displacements can be used to find strains according to (5) and stresses using (6).

\section{Conclusions}

The presented mathematical and numerical models of the three dimensional problem of thermoelasticity show the methodology of the Discontinuous Galerkin Method in the case of second order partial differential equations. On the basis of presented considerations a computer program can be easily created. Unfortunately, the number of iterations necessary to achieve reasonable accuracy of results can be very large, which drastically lengthens the calculation process compared to continuous approach. The advantage of DGM is that each finite element is independent thus the calculation process can be easily parallelized.

\section{References}

[1] Cockburn, B., Karniadakis, G.E., \& Shu, C.W. (2000). Discontinuous Galerkin Methods. Theory, Computations and Applications, vol. 11 of Lecture Notes in Computational Science and Engineering, Berlin: Springer.

[2] Lew, A., Neff, P., Sulsky, D., \& Ortiz, M. (2004). Optimal BV estimates for a discontinuous Galerkin method for linear elasticity. Applied Mathematics Research Express, 3, 73-106.

[3] Hansbo, P., \& Larson, M.G. (2002). Discontinuous Galerkin methods for incompressible and nearly incompressible elasticity by Nitsche's method. Computer Methods in Applied Mechanics and Engineering, 191(17-18), 1895-1908.

[4] Hansbo, P., \& Larson, M.G. (2003). Discontinuous Galerkin and the Crouzeix-Raviart element: Application to Elasticity. Mathematical Modelling and Numerical Analysis, 37(1), 63-72.

[5] Riviere, B. (2008). Discontinuous Galerkin methods for solving elliptic and parabolic equations: Theory and implementation. Frontiers in Mathematics 35, SIAM.

[6] Chen, Y., Huang, J., Huang, X., \& Xu Y. (2010). On the local discontinuous Galerkin method for linear elasticity. Mathematical Problems in Engineering, 20.

[7] Arnold, D.N., \& Winther, R. (2002). Mixed finite elements for elasticity. Numerische Mathematik, 92(3), 401-419.

[8] Adams, S., \& Cockburn, B. (2005). A mixed finite element method for elasticity in three dimensions. Journal of Scientific Computing, 25(3), 515-521.

[9] Li, B.Q. (2006). Discontinuous Finite Elements in Fluid Dynamics and Heat Transfer. London: Springer-Verlag. 